\title{
Oxymatrine exerts a protective effect in myocardial ischemia/reperfusion-induced acute lung injury by inhibiting autophagy in diabetic rats
}

\author{
ZHEN XIONG ${ }^{1 *}$, JIALI XU ${ }^{2 *}$ and $\mathrm{XIN} \mathrm{LIU}^{3}$ \\ Departments of ${ }^{1}$ Children's Health Care, ${ }^{2}$ Respiratory Medicine and ${ }^{3}$ Neonatology, Wuhan Children's Hospital, \\ Tongji Medical College, Huazhong University of Science and Technology, Wuhan, Hubei 430015, P.R. China
}

Received July 8, 2020; Accepted December 2, 2020

DOI: $10.3892 / \mathrm{mmr} .2021 .11822$

\begin{abstract}
Oxymatrine (OMT) is the primary active component of Sophora flavescens Ait., and is widely used for the treatment of diabetic complications. The present study aimed to investigate the effects of OMT on acute lung injury (ALI) in diabetic rats subjected to myocardial ischemia/reperfusion (I/R). ALI in a myocardial I/R model was established in streptozocin-induced diabetic rats. Enzyme-linked immunosorbent assays were used to evaluate the levels of creatine kinase isoenzyme MB and lactate dehydrogenase, and the inflammatory response was assessed via leukocyte counts and the levels of tumor necrosis factor (TNF)- $\alpha$, interleukin (IL)- 6 and IL-8 in the bronchoalveolar lavage (BAL) fluid. Hematoxylin and eosin staining was used to determine pathological changes to the lung tissue, and the autophagy-related proteins LC-3II/LC-3I, Beclin-1, autophagy protein 5 (Atg5) and p62 were detected by western blotting. Diabetic rats subjected to myocardial I/R showed increased levels of ALI with a higher lung injury score and WET/DRY ratio, and lower partial pressure of oxygen. This was accompanied by aberrant autophagy, indicated by an increased LC-3II/LC-3I ratio, decreased p62 expression levels, increased Atg5 and beclin-1 expression levels, decreased superoxide dismutase activity and increased 15-F2t-isoprostane formation in lung tissues, as well as increased levels of leukocytes, TNF- $\alpha$, IL-6 and IL- 8 in the BAL fluid. Administration of the autophagy inducer rapamycin significantly accelerated these alterations, while the autophagy inhibitor 3-Methyladenine exerted the
\end{abstract}

Correspondence to: Professor Xin Liu, Department of Neonatology, Wuhan Children's Hospital, Tongji Medical College, Huazhong University of Science and Technology, 100 Hongkong Road, Wuhan, Hubei 430015, P.R. China

E-mail: xinliuwch@163.com

*Contributed equally

Key words: diabetes, oxymatrine, inflammatory response, acute lung injury, ischemia/reperfusion opposite effects. These results indicated that diabetic lungs are more vulnerable to myocardial I/R, which was associated with aberrant autophagy. Furthermore, oxymatrine was observed to reverse and alleviate ALI in diabetic rats with myocardial $\mathrm{I} / \mathrm{R}$ in a concentration-dependent manner, the mechanism of which may be associated with the inhibition of autophagy.

\section{Introduction}

Type 2 diabetes mellitus (T2DM) is a long-term metabolic disorder characterized by a reduction in insulin secretion and an increase in insulin resistance. T2DM is regarded as one of the most common chronic diseases that can advance to various organ-associated complications, resulting in an increase in morbidity and mortality. Persistent glycemic dysfunction promotes numerous pathological changes and causes functional damage to organs, including the kidney, eye, nervous and cardiovascular systems (1). Chronic diabetes may also promote pulmonary dysfunction, and widespread attention surrounds the concept of 'diabetic lungs' (2). Epidemiological, experimental and clinical evidence has suggested that high glucose-induced oxidative stress and inflammation are implicated in the diabetic lung, and accelerate the decline in respiratory function $(3,4)$. Several studies have also demonstrated the subclinical significance of the diabetic lung, and that patients with diabetes are prone to severe respiratory disorders in the event of acute or chronic lung and/or cardiac disease $(2,5)$. Further clinical and experimental data have indicated that inflammatory responses and metabolic abnormalities in diabetes aggravate the acute lung injury (ALI) via autophagy $(6,7)$. Myocardial infarction is considered to be one of the more common complications underlying diabetes-associated mortality. Myocardial ischemia/reperfusion (I/R) has been reported to promote the injury of distant organs, including the lung $(8,9)$. Further studies have confirmed that ALI caused by myocardial I/R is more severe in patients with diabetes than in those without diabetes (10). However, there are few reports concerning the potential underlying mechanisms of ALI in association with myocardial I/R, and under diabetic conditions.

Autophagy is a catabolic cellular process that conservatively maintains cell homeostasis and survival. The key phases 
of autophagy, such as autophagosome biogenesis, lysosomal fusion and product degradation, are collectively termed 'autophagy flux', which is elevated during ischemia and markedly enhanced following tissue reperfusion. Inhibiting excessive autophagy may alleviate tissue damage under various pathological conditions. Numerous studies have demonstrated that autophagic dysfunction is closely associated with diabetic complications $(11,12)$. A significant time-related effect of lung injury has been associated with the diabetic condition, demonstrating the involvement of autophagy in ALI caused by pulmonary I/R in patients with diabetes (13). Another study suggested that pulmonary I/R impairment of the autophagic state, and a moderate increase in autophagy-associated injury, are beneficial for attenuating lung I/R injury (14). Collectively, these findings suggest that maintaining a moderate level of autophagy may help to reduce I/R-induced lung injury and promote cell survival in vivo. However, in diabetes, the role of autophagy in ALI caused by myocardial I/R has not been fully elucidated.

Oxymatrine (OMT) is a quinolizidine alkaloid derived from Sophora flavescens Ait., which is commonly used to treat a variety of pathological states, including inflammation, fibrosis and autophagy. OMT has been used to treat inflammatory diseases, damage to the heart caused by myocardial ischemia (15) and hypertension (16), and previous studies have demonstrated its protective effects against I/R injury (17). The underlying mechanism by which OMT operates may be associated with the inhibition of oxidative stress and inflammation, and the induction of apoptosis. However, there are limited relevant reports concerning the effect of OMT on autophagy in myocardial I/R-associated ALI in diabetic rats. Therefore, the present study may improve our understanding of how OMT ameliorates autophagy in myocardial I/R-induced ALI under diabetic conditions.

\section{Materials and methods}

Animal experiments. A total of 100 male Sprague-Dawley rats (weight, 250-300 g) were purchased from the Hunan SJA Laboratory Animal Co., Ltd. The animals were maintained under a $12 \mathrm{~h}$ light/dark cycle at $22-25^{\circ} \mathrm{C}$, with $50-65 \%$ humidity. All animals had free access to food and water. The present study was performed following the Guide for the Care and Use of Laboratory Animals, published by the National Institutes of Health (NIH publication no. 85-23, revised in 1996) (18), and the experiments were approved by the Huazhong University of Science and Technology Medicine Animal Care and Use Committee (Wuhan, China), according to the regulation of the study of pain, and the Guide for the Care and Use of Laboratory Animals.

Reagents and antibodies. OMT was purchased from Xi'an Aladdin Biological Technology Co., Ltd., (cat. no. 16837-52-8) and dissolved in PBS. Assay kits for the assessment of blood glucose, cytokines [tumor necrosis factor (TNF)- $\alpha$ (cat. no. H052), interleukin (IL)-6 (cat. no. H007) and IL-8 (cat.no.H008; Beyotime Institute of Biotechnology)], leukocyte counts, myocardial enzymes [lactate dehydrogenase (LDH) (cat.no. A020-2-2) and creatine kinase isoenzyme MB (CK-MB; cat. no. H197)], superoxide dismutase (SOD; cat. no. A001-1-2;) and 15-F2t-isoprostane (15-F2t-IsoP; cat. no. A151-1-1) were purchased from Nanjing Jiancheng Bioengineering Institute. Antibodies against LC3I (cat. no. 4108), LC3II (cat. no. 3868), beclin-1 (cat. no. 4122), p62 (cat. no. 48768) and autophagy protein 5 (Atg5; cat. no. 9980) were purchased from Cell Signaling Technology, Inc. Anti- $\beta$-actin (cat. no. BM3873), as well as anti-rabbit (cat. no. BA1041) and anti-mouse secondary antibodies (cat. no. BM2020) were purchased from Wuhan Boster Biological Technology, Ltd. The autophagy inhibitor 3-Methyladenine (3-MA; cat. no. M9281) and the autophagy inducer rapamycin (Rap; cat. no. V900930) were purchased from Sigma-Aldrich (Merck KGaA).

Induction and assessment of diabetes. Male Sprague-Dawley rats were randomly divided into following groups: i) Control group $(n=8)$; ii) sham group (surgery with no ischemia) $(n=8)$; and iii) diabetes mellitus (DM) group $(n=40)$. DM group were fed a high-glucose, high-fat diet consisting of $70 \%$ standard laboratory chow, 15\% carbohydrate, $10 \%$ lard and $5 \%$ yolk powder. After 4 weeks, the rats received a single intraperitoneal injection of streptozocin (STZ, $35 \mathrm{mg} / \mathrm{kg}$ ) and the high-glucose, high-fat diet was continued. At week 8, blood glucose was assessed using a sample from the tail vein; a fasting blood glucose level $\geq 7.0 \mathrm{mmol} / 1$, or a random blood glucose level $\geq 11.0 \mathrm{mmol} / 1$, was defined as diabetic, and these rats were selected for further investigation $(n=40)$.

Establishment of myocardial I/R injury. The coronary artery ligation method was used to establish the myocardial I/R injury model. Control and DM rats were anesthetized with an intraperitoneal injection of sodium pentobarbital $(30 \mathrm{mg} / \mathrm{kg})$, and subsequently received endotracheal intubation and artificial ventilation. Following exposure of the heart, a thread was passed through the left coronary artery, and another two threads were drawn from the knot to loosen the ligature. The left coronary artery was ligated to induce ischemia, resulting in a cyanotic appearance to the local myocardium. After $1 \mathrm{~h}$ of ischemic induction, the ligature was loosened to restore blood flow and initiate reperfusion, which was continued for an additional $1 \mathrm{~h}$.

The DM group were randomly divided into eight groups as follows ( $n=8$ per group): i) Sham group, surgery with no ischemia, DM without ischemia; ii) I/R model group, DM and myocardial I/R; iii) I/R+OMT (12.5 mg/kg) group, DM and myocardial I/R with $12.5 \mathrm{mg} / \mathrm{kg} \mathrm{OMT}$; iv) IR+OMT (25 mg/kg) group, DM and myocardial I/R with $25 \mathrm{mg} / \mathrm{kg}$ OMT; v) IR+OMT (50 mg/kg) group, DM and myocardial I/R with $50 \mathrm{mg} / \mathrm{kg}$ OMT; vi) I/R+Rap group, DM and myocardial I/R with $15 \mathrm{mg} / \mathrm{kg}$ Rap; and vii) I/R+3-MA group, DM and myocardial I/R with $10 \mathrm{mg} / \mathrm{kg} 3-\mathrm{MA}$. OMT was dissolved in isotonic saline according to previous reports $(13,14)$, and the rats were administered OMT by gavage $10 \mathrm{~min}$ prior to occlusion of the left coronary artery. After $25 \mathrm{~min}$ of reperfusion, Rap and 3-MA were administered to the appropriate groups to compare the effects of these compounds on I/R. At the end of the study, rats were anesthetized by an intraperitoneal injection of pentobarbital sodium $(30 \mathrm{mg} / \mathrm{kg})$ until they lost consciousness. Blood samples $(5 \mathrm{ml})$ were collected from the abdominal aorta for subsequent analysis. All the animals were sacrificed following anesthesia by exsanguination, and 
their heart and lung tissues were collected for further experiments.

Blood glucose detection. Blood samples were obtained from the abdominal aorta, allowed to clot for $30 \mathrm{~min}$ at $4^{\circ} \mathrm{C}$, and centrifuged at $3,500 \mathrm{xg}$ for $10 \mathrm{~min}\left(4^{\circ} \mathrm{C}\right)$. The supernatant was used to assess the level of blood glucose, which was determined using a commercially available glucose kit.

Blood gas analysis, WET/DRY ratio, and hematoxylin and eosin $(H \& E)$ staining. Carotid blood gas analysis [partial pressure of oxygen $\left(\mathrm{PO}_{2}\right)$ ] was conducted following $2 \mathrm{~h}$ of reperfusion; the WET/DRY ratio and H\&E staining were used to assess the degree of pulmonary injury. The lung tissues were weighed and dried for $48 \mathrm{~h}$ in an oven at a constant temperature of $60^{\circ} \mathrm{C}$; when a dehydrated consistency was achieved, the tissues were weighed once again. The twice weight ratio was calculated as an indicator of edema. Specimens from the left lower lobe of the lung were fixed at $25^{\circ} \mathrm{C}$ in $4 \%$ paraformaldehyde for $24 \mathrm{~h}$, sectioned $(2-\mu \mathrm{m}$ thick) and stained with hematoxylin for $2 \mathrm{~min}$ at $25^{\circ} \mathrm{C}$ followed by eosin for $2 \mathrm{~min}$ at $25^{\circ} \mathrm{C}$. Light microscopic examination was conducted to determine the following: i) Pulmonary interstitial edema; ii) airway epithelial injury; iii) the degree of hyalinization; and vi) the lung injury score. The criteria for the lung injury score was membrane formation, neutrophil infiltration and alveolar hemorrhage $(7,19)$. The indices were graded as follows: i) Normal $=0$ '; ii) minimal change $=1$ '; iii) mild change $=2$ '; iv) moderate change $=3$ '; and v) severe change $=4$ '. Collectively, each section received five scores, and the scores for each criterion were used to evaluate the degree of lung tissue injury.

Detection of myocardial enzyme levels. Following sacrifice, the blood of each rat was collected via the tail vein and the serum was isolated as aforementioned. Using commercially available kits (per the manufacturers' instructions), LDH and CK-MB were detected to evaluate the level of damage to the heart muscle.

Determination of SOD, GSH and 15-F2t-IsoP levels. Lung tissues were homogenized, centrifuged at $4,000 \times \mathrm{g}, 4^{\circ} \mathrm{C}$ for $10 \mathrm{~min}$, and the supernatant was collected and stored at $-80^{\circ} \mathrm{C}$. The levels of SOD, GSH and 15-F2t-IsoP were determined using commercially available kits per the manufacturers' protocols.

Detection of leukocyte count, and the levels of TNF- $\alpha, I L-6$ and IL-8 in the bronchoalveolar lavage (BAL) fluid. The leukocyte count, and the levels of TNF- $\alpha$, IL- 6 and IL- 8 in the BAL fluid of diabetic rats were evaluated using commercially available kits.

Western blotting. Total protein was extracted from lung tissues using RIPA buffer (containing 0.1\% PMSF; cat. no. R0278; Sigma-Aldrich; Merck KGaA). Equal amounts of protein from each sample $(50 \mathrm{mg})$ were separated via SDS-PAGE on $10 \%$ or $12 \%$ gels, and subsequently transferred to polyvinylidene fluoride membranes. The membranes were then incubated with primary antibodies overnight at $4^{\circ} \mathrm{C}$, and washed prior to a second incubation with horseradish peroxidase (HRP)-coupled goat anti-mouse $(1: 1,000)$ or goat anti-rabbit secondary antibodies $(1: 1,000)$ for $1 \mathrm{~h}$ at room temperature. The chemiluminescence signals were detected using the EasySee ${ }^{\circledR}$ Western Blot Kit (Beijing TransGen Biotech Co., Ltd.), and densitometric analysis was conducted using ImageJ 1.43 (National Institutes of Health). The primary antibodies and their dilutions were as follows: Anti-LC3I $(1: 1,000)$, LC3II $(1: 1,000)$, beclin-1 (1:1,000), p62 (1:1,000), Atg5 (1:1,000) and $\beta$-actin (1:1,000; cat. no. BM3873; Wuhan Boster Biological Technology, Ltd).

Statistical analysis. SPSS 21.0 (IBM Corp.) was used for statistical analysis, and all data are presented as the mean \pm standard deviation (SD). For gene expression, one-way ANOVA followed by Tukey's post hoc test was used to test for differences among groups. To analyze lung injury scores, Kruskal-Wallis test was performed, followed by Dunn's multiple comparison test. $\mathrm{P}<0.05$ was considered to indicate a statistically significant difference.

\section{Results}

Myocardial injury following myocardial $I / R$ in diabetic rats. Following myocardial I/R, the morphology of the myocardial tissue of diabetic rats was notably altered; myocardial fiber arrangement was disordered and even partially dissolved (Fig. 1A). Furthermore, the levels of CK-MB and LDH, markers of myocardial tissue damage, were significantly higher in the $\mathrm{DM}+$ myocardial $\mathrm{I} / \mathrm{R}$ group than those in the Sham group $(\mathrm{P}<0.05$; Fig. $1 \mathrm{~B}$ and $\mathrm{C})$. These findings suggested that hyperglycemic rats are prone to myocardial injury, and that I/R accelerates diabetic myocardial injury.

Diabetes aggravates ALI following myocardial I/R, which is accompanied by an impaired autophagy status. Present experimentation demonstrated that ALI occurs in diabetic rats following myocardial I/R. As shown in Fig. 2A-D, H\&E staining of the lung tissues revealed ALI with alveolar and interstitial edema, hemorrhage and inflammatory cell infiltration. These pathological changes were more severe in diabetic rats. Compared with the Sham group, the degree of lung injury in the DM+myocardial I/R groups was significantly increased compared with the control and sham groups $(\mathrm{P}<0.05$; Fig. $2 \mathrm{E})$. These results indicated that diabetic rats with myocardial $I / R$ exhibited a greater degree of lung injury than those in the Sham group.

Effects of Rap and 3-MA on ALI following myocardial I/R in diabetic rats. As shown in Fig. 3, compared with the Sham group, myocardial I/R significantly decreased lung function in rats $(\mathrm{P}<0.05$; Fig. 3A); lung tissue damage was significantly enhanced $(\mathrm{P}<0.05$; Fig. $3 \mathrm{~B})$, and the WET/DRY ratio of the lung tissue was increased $(\mathrm{P}<0.05$; Fig. $3 \mathrm{C})$, indicating that myocardial I/R injury induces and accelerates ALI in diabetic rats. The autophagy inducer Rap further enhanced I/R-induced ALI damage, and by contrast, the inhibitor 3-MA alleviated ALI compared with the $\mathrm{DM}+$ myocardial I/R group $(\mathrm{P}<0.05$; Fig. 3A-C).

Autophagy is reportedly initiated by the activation of beclin-1 and the binding of LC-3II to autophagosomes. 


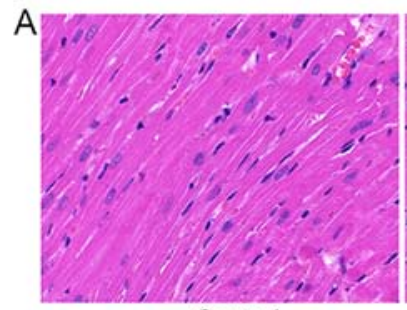

Control

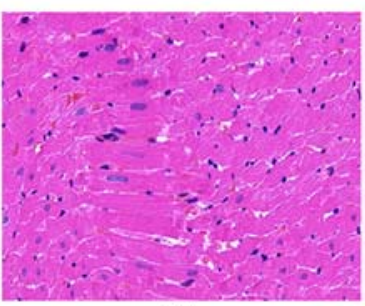

$\mathrm{DM}$

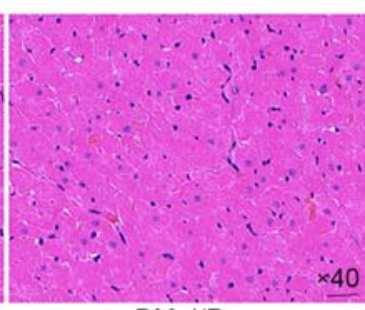

$\mathrm{DM}+\mathrm{l} / \mathrm{R}$
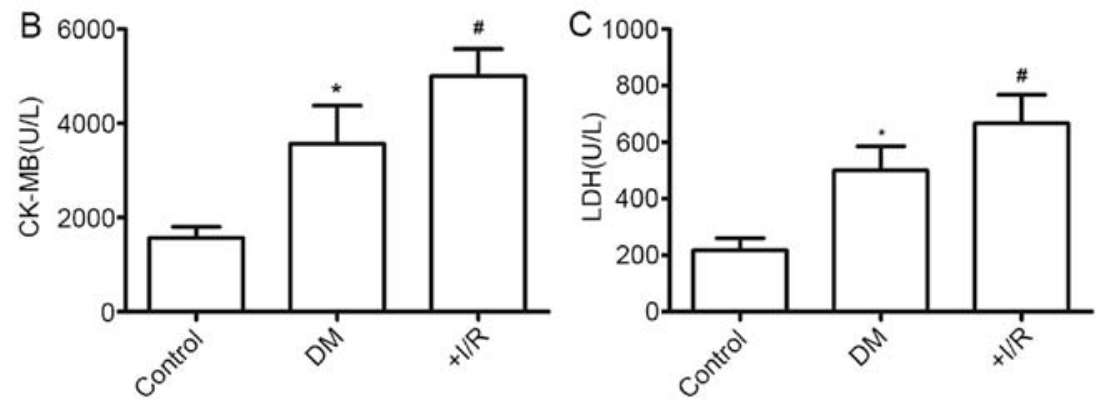

Figure 1. Myocardial injury induced by myocardial I/R in DM rats. (A) Histopathological changes in the lungs of control and DM rats subjected to sham surgery or myocardial I/R were observed using light microscopy (magnification, $\mathrm{x} 40$ ). It was observed that the arrangement of myocardial fibers was disordered, and some were no longer visible. Expression levels of (B) CK-MB and (C) LDH. Values are expressed as the mean \pm SD from three independent experiments. ${ }^{*} \mathrm{P}<0.05$ and ${ }^{\#} \mathrm{P}<0.01$ vs. the Sham group. I/R, ischemia/reperfusion; CK-MB, creatine kinase isoenzyme MB; LDH, lactate dehydrogenase; DM, diabetes mellitus.
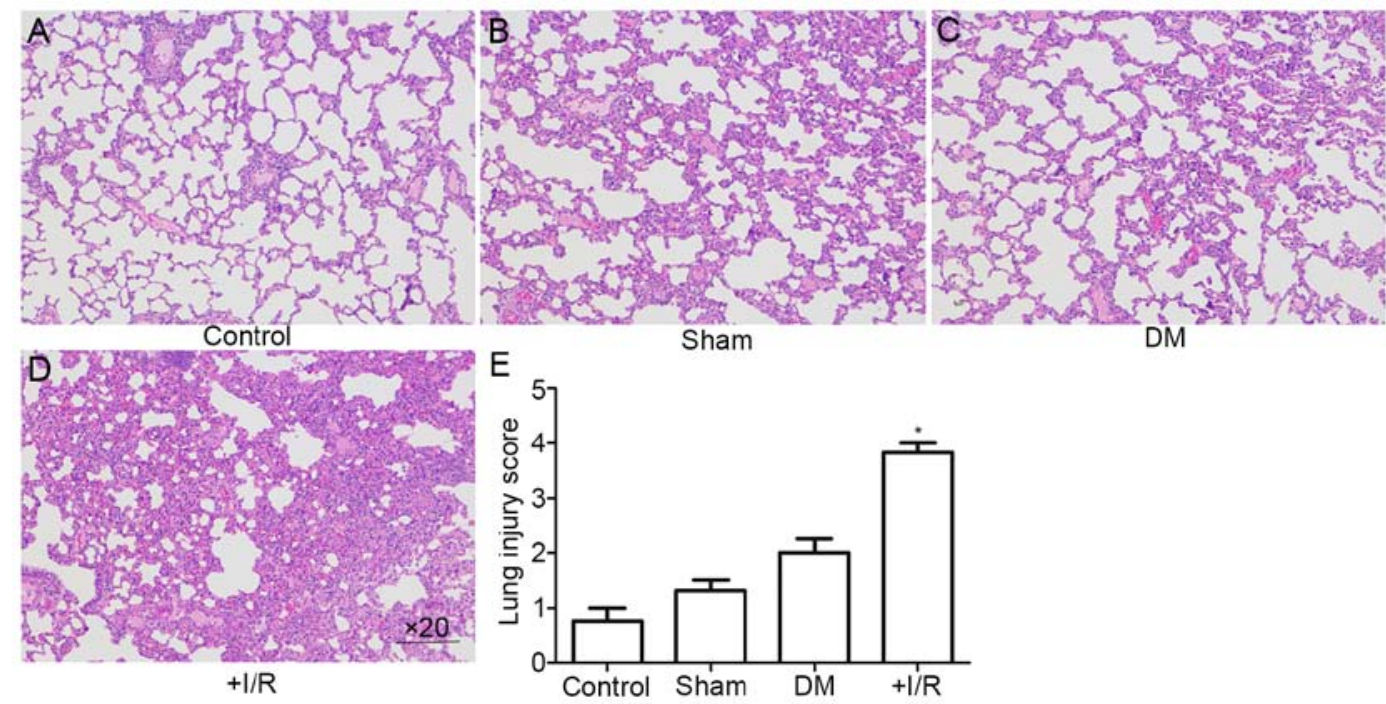

Figure 2. Diabetes aggravates acute lung injury following myocardial I/R. Histopathological changes in the (A) control, (B) Sham, (C) DM and (D) DM+I/R groups. Acute lung injury with interalveolar and interstitial edema, bleeding and inflammatory cell infiltration were observed using light microscopy (magnification, $\mathrm{x} 20$ ). (E) Lung injury score was quantified. All values are expressed as the mean $\pm \mathrm{SD}, \mathrm{n}=8$. * $\mathrm{P}<0.05$ vs. the control group. DM, diabetes mellitus; $\mathrm{I} / \mathrm{R}$, ischemia/reperfusion.

Thus, beclin-1 and LC-3II expression are considered as specific markers of autophagic initiation (20). As shown in Fig. 3D and E, the expression levels of LC-3II/LC-3I and beclin-1 were significantly increased in diabetic rats compared with those in the Sham group $(\mathrm{P}<0.05)$, which was enhanced by Rap and reversed by 3-MA treatment. These results indicated that diabetes accompanied by myocardial I/R impairs the autophagic state.

Effects of Rap and 3-MA on pulmonary inflammation and oxidative stress in diabetic rats subjected to myocardial $I / R$. Myocardial I/R aggravates inflammation and oxidative stress in diabetic rats, which promotes autophagy-associated injury. The leukocyte count, and the levels of TNF- $\alpha$, IL- 6 and IL-8 have been regarded as common indicators of the extent of lung injury. Thus, the BAL fluid was collected and analyzed to assess the severity of ALI in diabetic rats. As shown in Fig. 4A-D, the leukocyte counts and levels of TNF- $\alpha$, IL- 6 and IL-8 in the BAL fluid of diabetic rats were significantly higher than those in the Sham group $(\mathrm{P}<0.05)$. These results suggested a severe inflammatory reaction in diabetic rats following myocardial I/R. The autophagy inducer Rap significantly increased the leukocyte count and levels of inflammatory cytokines, whereas the autophagy inhibitor 3-MA had the opposite 
A

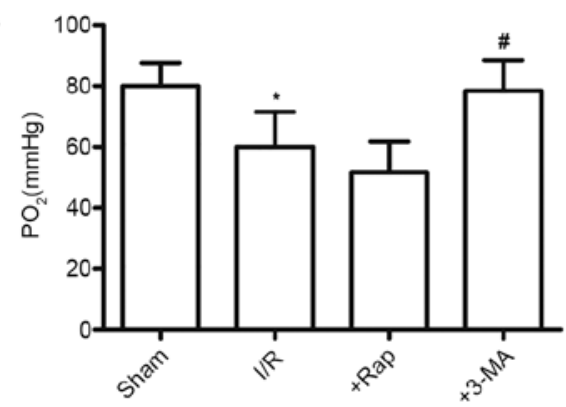

C

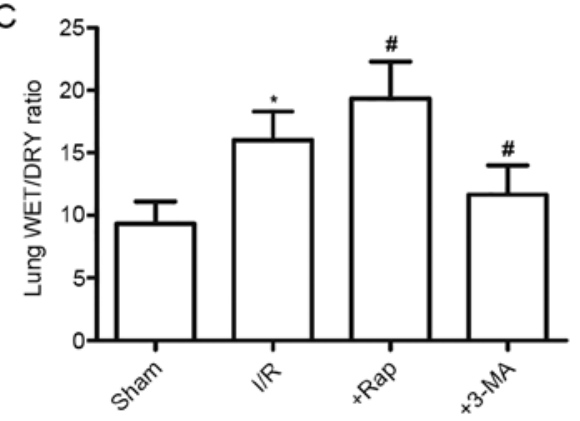

E

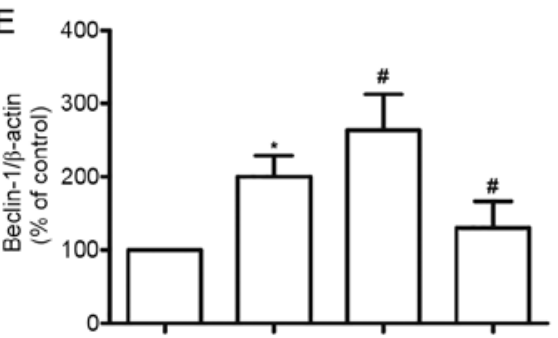

Beclin-1

$\beta$-actin

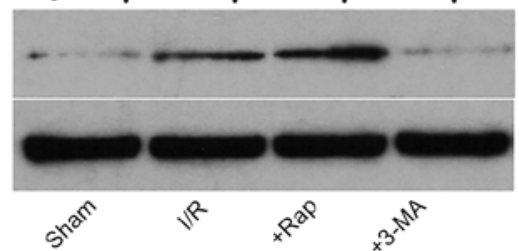

B

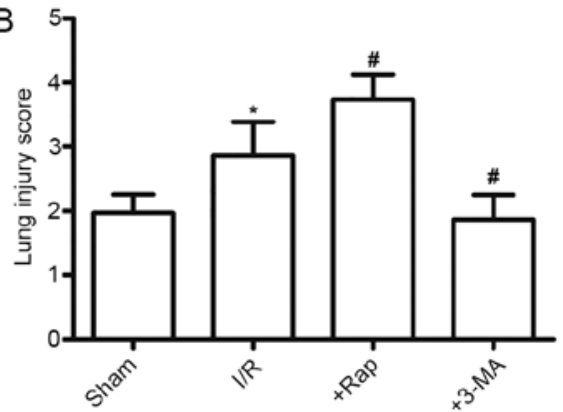

D
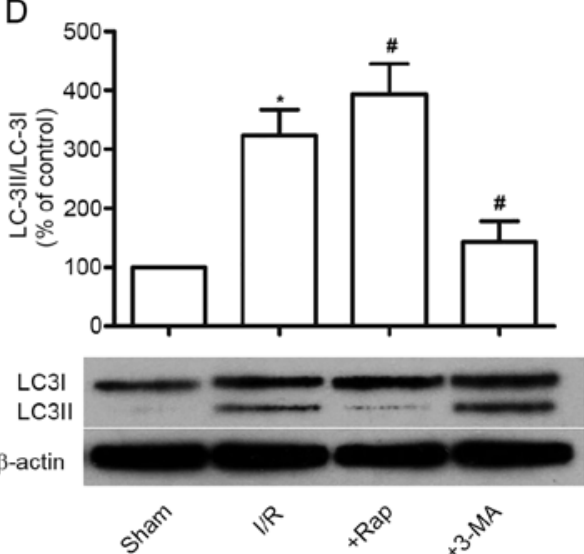

Figure 3. Effects of Rap and 3-MA on acute lung injury following myocardial I/R in DM rats. Effects of Rap and 3-MA on (A) carotid blood PO ${ }_{2}$, (B) lung injury score and (C) WET/DRY ratio. Representative western blot images demonstrating the effects of Rap and 3-MA on (D) LC-3II/LC-3I and (E) Beclin-1 expression levels in control and $\mathrm{DM}$ rats following myocardial I/R. All values are expressed as the mean $\pm \mathrm{SD}, \mathrm{n}=8$. ${ }^{*} \mathrm{P}<0.05$ vs. the $\mathrm{Sham}$ group. ${ }^{\#} \mathrm{P}<0.05$ vs. I/R group. Rap, rapamycin; 3-MA, 3-Methyladenine; I/R, ischemia/reperfusion; $\mathrm{PO}_{2}$, partial pressure of oxygen; DM, diabetes mellitus.

effects $(\mathrm{P}<0.05)$. The influence of autophagy on pulmonary oxidative stress in diabetic rats was also investigated following myocardial I/R. The results also showed that ALI significantly decreased SOD activity and increased 15-F2t-IsoP formation, as compared with the Sham group ( $\mathrm{P}<0.05$; Fig. 4E and F). All pulmonary inflammatory changes were increased by Rap, but were reversed by 3-MA administration.

Effect of OMT on autophagy in myocardial I/R-induced lung injury in diabetic rats. Autophagy is activated when myocardial I/R in diabetic rats induces ALI. To investigate the effect of OMT on autophagy in myocardial I/R-induced ALI in diabetic rats, the expression of LC-3II/LC-3I, Beclin-1, p62 and Atg5 was evaluated. Compared with the Sham group, the expression levels of LC-3II/LC-3I, Beclin-1 and Atg5 were significantly upregulated, and the expression of p62 was downregulated following myocardial $I / R$ in diabetic rats $(P<0.05$; Fig. 5A-D). Furthermore, these effects were reversed following treatment with OMT, in a dose-dependent manner $(\mathrm{P}<0.05)$. These findings suggested that OMT inhibits ALI-induced autophagy in diabetic rats.

\section{Discussion}

At present, there are an increasing number of studies focusing on diabetic complications, including those of the heart and kidney, as well as retinopathic injuries $(21,22)$. However, relatively few reports have focused on the influence of diabetes on the lungs, especially in ALI induced by diabetic myocardial infarction. The results of the present study revealed that the diabetic lung is more susceptible to myocardial I/R, and provided further evidence that ALI following myocardial I/R is associated with aberrant autophagy. Using the autophagy inducer Rap or the autophagy inhibitor 3-MA, it was further confirmed that autophagy is implicated in ALI caused by myocardial I/R under diabetic conditions, which is consistent 

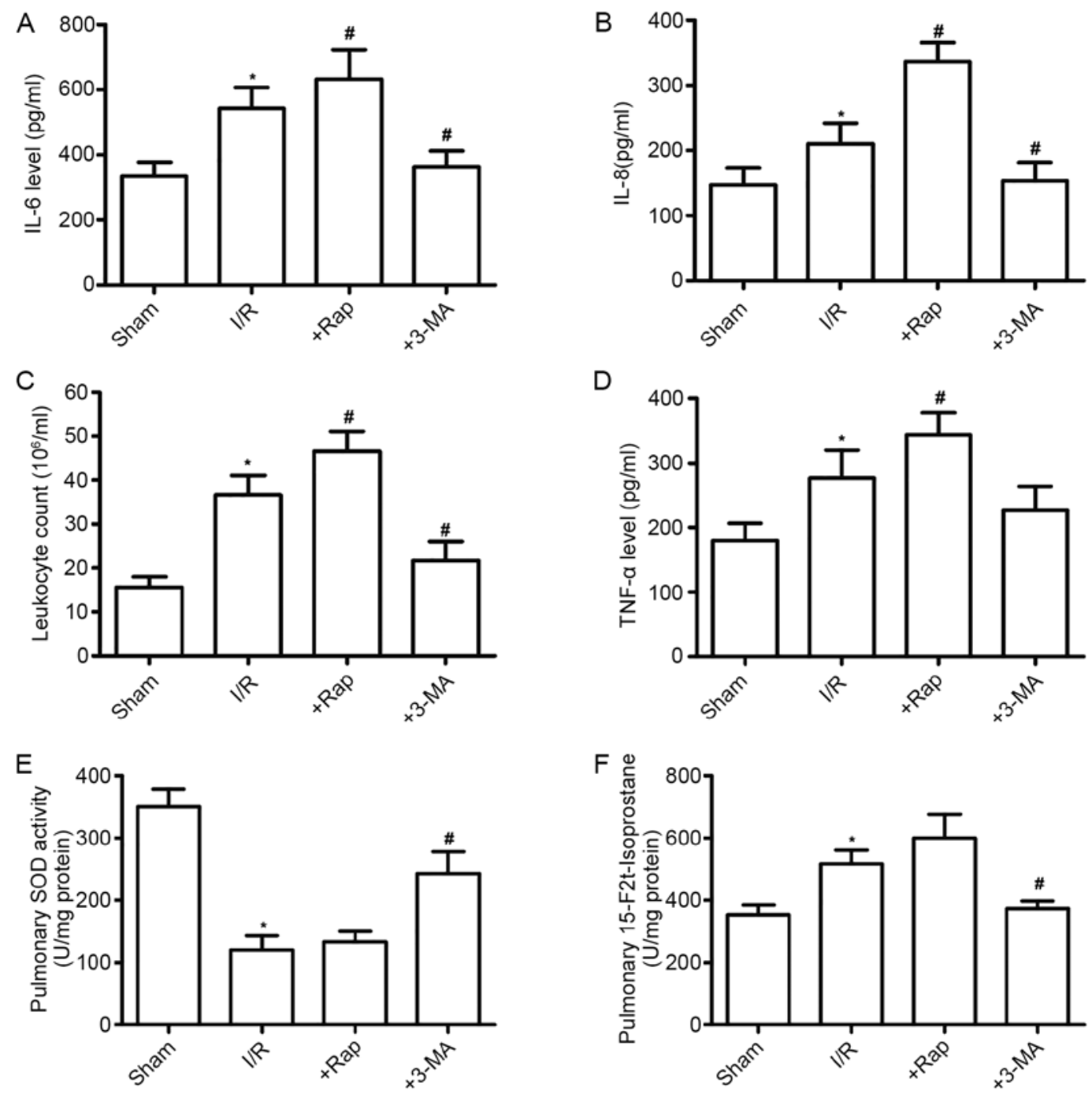

Figure 4. Effects of Rap and 3-MA on pulmonary inflammation in DM rats subjected to myocardial I/R. Effects of Rap and 3-MA on (A) IL-6, (B) IL-8, (C) leukocyte counts and (D) TNF- $\alpha$ in the bronchoalveolar lavage fluid. Effects of Rap and 3-MA on (E) SOD and (F) 15-F2t-Isop in lung tissues from control and DM rats following myocardial I/R. All values are expressed as the mean $\pm \mathrm{SD}, \mathrm{n}=8$. ${ }^{*} \mathrm{P}<0.05$ vs. the Sham group; ${ }^{*} \mathrm{P}<0.05$ vs. the I/R group. Rap, rapamycin; 3-MA, 3-Methyladenine; I/R, ischemia/reperfusion; IL, interleukin; TNF- $\alpha$, tumor necrosis factor- $\alpha$; SOD, superoxide dismutase; 15 -F2t-Isop, 15-F2t-isopro stane; DM, diabetes mellitus.

with previous findings $(5,7)$. To the best of our knowledge, the present study was the first to investigate the protective effects of OMT on myocardial I/R-associated ALI, via the inhibition of autophagy in diabetic rats.

Diabetes remains a major global health issue, and persistent hyperglycemia causes systemic damage to various organs; such complications include diabetic cardiomyopathy, diabetic nephropathy, retinopathy, neuropathy and diabetic foot $(1,23)$. Changes in the normal anatomy and biological characteristics of the lung during diabetes significantly accelerates the deterioration in respiratory function (2). Previous studies have demonstrated that diabetes disrupts ventilation by reducing respiratory muscle strength (24). Additionally, abnormal saccharification leads to thickening of the basement membrane and proliferation of vascular smooth muscle cells, reducing ventilation capacity and blood flow to the area. Experiencing acute or chronic lung and/or heart disease during diabetes confers vulnerability to the diabetic lung, and patients with diabetes are at a higher risk of developing cardiovascular diseases, and thus have a poor prognosis following myocardial infarction $(25,26)$. Acute myocardial ischemia is regarded to be the primary cause of ALI, resulting in acute severe pulmonary hypertension (27) and pulmonary hemodynamics (28). Previous studies have demonstrated several mechanisms of ALI that are associated with the pulmonary dysfunction induced by myocardial I/R $(29,30)$. Under diabetic conditions, the lung tissue is characterized by a large number of oxygen free radicals (primarily reactive oxygen species) accompanied by a decrease in its antioxidant capacity, including activation of NADPH oxidase, decreased SOD activity and reduced GSH levels. In addition, diabetes promotes the release of various proinflammatory cytokines, which further exacerbate reduced lung function and ALI. Numerous studies have demonstrated that inhibiting the pulmonary inflammatory response and reducing oxidative stress alleviates lung injury in diabetic conditions $(31,32)$. In vivo animal experiments have also indicated that myocardial I/R aggravates lung tissue injury, which is involved in oxidative stress and the inflammatory response in diabetes (33). The present study demonstrated that oxidative damage and inflammation accelerate diabetic lung 

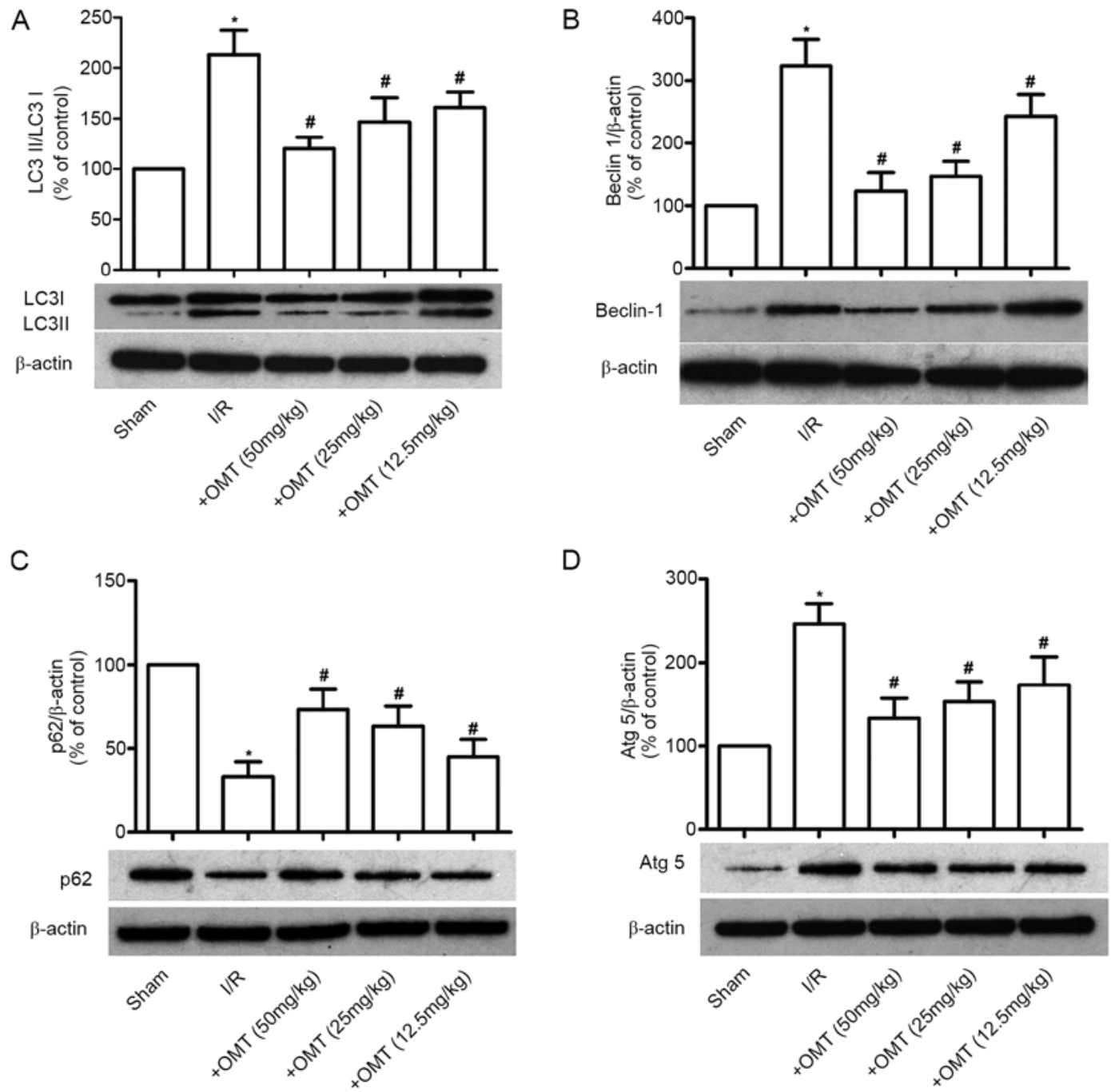

Figure 5. Effect of OMT on autophagy in lung injury after myocardial I/R in DM rats. Effects of OMT on (A) LC-3II/LC-3I, (B) Beclin-1, (C) p62 and (D) Atg5 expression levels. All values are expressed as the mean $\pm \mathrm{SD}, \mathrm{n}=8$. ${ }^{*} \mathrm{P}<0.05$ vs. the Sham group; " $\mathrm{P}<0.05$ vs. the I/R group. OMT, oxymatrine; $\mathrm{I} / \mathrm{R}$, ischemia/reperfusion; DM, diabetes mellitus; Atg5, autophagy protein 5.

injury, especially when accompanied by myocardial I/R. The results of the present study also revealed a significant decrease in the antioxidant capacity of lung tissue (including decreased SOD activity and increased formation of 15-F2t-IsoP), and an increase in inflammatory cytokines (leukocyte count, TNF- $\alpha$, IL-6 and IL-8) in diabetic rats, accompanied by higher lung injury scores and WET/DRY ratios, and a lower PO2; these findings are consistent with those previously reported (10), and indicate that myocardial I/R accelerates the loss of pulmonary function and results in severe lung injury in diabetic rats.

Autophagy is a eukaryotic cellular self-degradation and recycling process in which intracellular membrane structures package protein complexes and organelles to degrade and recycle intracellular proteins and damaged organelles, maintaining the normal cellular function. Under normal circumstances, autophagy is a homeostatic mechanism for cell survival and maintenance via the lysosomal degradation of cytoplasmic constituents. However, accumulating evidence indicates that under pathological conditions, such as inflammation, diabetes, a high-fat diet and oxidative stress, autophagic dysfunction is linked to cell death processes $(34,35)$. Autophagy has been demonstrated to play an important role in regulating normal function of pancreatic $\beta$ cells and insulin-target tissues in the liver, and adipose tissue. Enhanced autophagy also acts as a protective mechanism against oxidative stress in these tissues. Altered autophagic activity has been implicated in the progression of obesity to T2DM through impaired $\beta$ cell function and development of insulin resistance (36). Moreover, there is ample evidence to suggest that lung I/R injury promotes excessive autophagy (13), and cold ischemia preservation for lung transplantation has been shown to induce autophagy to varying degrees over different time periods (37). Based on this evidence, it was speculated that the severity and duration of I/R could influence the degree of autophagy, promoting either cell survival or death. The results of the present study further confirmed the fact that the pulmonary autophagy state was excessive in both control and diabetic rats subjected to $30 \mathrm{~min}$ ischemia, followed by $2 \mathrm{~h}$ reperfusion. Defective autophagy was also observed in lung tissues subjected to I/R, which is consistent with a previous report (16). The expression levels of markers of autophagy, such as of LC3-II/LC3-I and Beclin-1 were therefore investigated in the present study, and were found to be increased in diabetic rats following myocardial I/R, suggesting that myocardial I/R induces autophagy. 
Furthermore, an upregulation in Atg5 and a downregulation in p62 were observed in the diabetic rats, and these effects were reduced by OMT. Autophagy selectively degrades damaged cellular organelles and protein aggregates, while apoptosis removes damaged or aged cells. Maintaining a balance between autophagy and apoptosis is critical for cell fate. I/R accelerates autophagy and then contributes to cell apoptosis, which is widely accepted. In present study, it was found that I/R disturbed the balance between autophagy and cell apoptosis, which exacerbated tissue damage.

In order to clarify whether diabetes induced by STZ or a high-sugar, high-fat diet promotes autophagy, the phosphoinositide 3-kinase inhibitor, 3-MA, was utilized. Rap was also used to induce autophagy. During treatment with OMT, LC3-II expression was significantly decreased in diabetic rats in a concentration-dependent manner. The effect of OMT was similar to that of 3-MA. To the best of our knowledge, the present study is the first to demonstrate that OMT ameliorates myocardial I/R-induced ALI by inhibiting autophagy in diabetic rats.

In conclusion, the present study demonstrated that diabetes-impaired autophagic status is associated with ALI following myocardial I/R in diabetic rats. Blocking autophagy may improve myocardial I/R-induced ALI by inhibiting inflammatory and oxidative stress responses. Furthermore, OMT inhibits ALI-associated autophagy following diabetic myocardial I/R. These results suggested that OMT treatment may be an effective strategy for maintaining autophagic homeostasis.

\section{Acknowledgements}

Not applicable.

\section{Funding}

The present study was supported by Hubei National Science Funds (grant no. 2017JJ2343).

\section{Availability of data and materials}

The datasets used and/or analyzed during the current study are available from the corresponding author on reasonable request.

\section{Authors' contributions}

ZX designed the study, performed the experiments, and analyzed, interpreted and presented results for group discussions. ZX, XL and JX designed the study, performed the experiments and wrote the manuscript. XL provided rationale, background, framework and feedback. All authors read and approved the final manuscript.

\section{Ethics approval and consent to participate}

The present study was performed following the Guide for the Care and Use of Laboratory Animals, published by the National Institutes of Health (NIH publication no. 85-23, revised in 1996), and the experiments were approved by the Huazhong University of Science and Technology Medicine Animal Care and Use Committee (Wuhan, China; approval no. 2019-0013), according to the regulation of the study of pain, and the Guide for the Care and Use of Laboratory Animals.

\section{Patient consent for publication}

Not applicable.

\section{Competing interests}

The authors declare that they have no competing interests.

\section{References}

1. Gregg EW, Sattar N and Ali MK: The changing face of diabetes complications. Lancet Diabetes Endocrinol 4: 537-547, 2016.

2. Pitocco D, Fuso L, Conte EG, Zaccardi F, Condoluci C, Scavone G, Incalzi RA and Ghirlanda G: The diabetic lung-a new target organ? Rev Diabet Stud 9: 23-35, 2012.

3. Anhê FF, Roy D, Pilon G, Dudonné S, Matamoros S, Varin TV Garofalo C, Moine Q, Desjardins Y, Levy E and Marette A: A polyphenol-rich cranberry extract protects from diet-induced obesity, insulin resistance and intestinal inflammation in association with increased Akkermansia spp. population in the gut microbiota of mice. Gut 64: 872-883, 2015.

4. Khateeb J, Fuchs E and Khamaisi M: Diabetes and lung disease: A neglected relationship. Rev Diabet Stud 15: 1-15, 2019.

5. Yeh F, Dixon AE, Marion S, Schaefer C, Zhang Y, Best LG, Calhoun D, Rhoades ER and Lee ET: Obesity in adults is associated with reduced lung function in metabolic syndrome and diabetes: The strong heart study. Diabetes Care 34: 2306-2313, 2011.

6. Li K, Li M, Li W, Yu H, Sun X, Zhang Q, Li Y, Li X, Li Y, Abel ED, et al: Airway epithelial regeneration requires autophagy and glucose metabolism. Cell Death Dis 10: 875, 2019.

7. Zhan L, Zhang Y, Su W, Zhang Q, Chen R, Zhao B, Li W, Xue R, $\mathrm{Xia} Z$ and Lei $\mathrm{S}$ : The roles of autophagy in acute lung injury induced by myocardial ischemia reperfusion in diabetic rats. J Diabetes Res 2018: 5047526, 2018.

8. Wang Y, Ji M, Chen L, Wu X and Wang L: Breviscapine reduces acute lung injury induced by left heart ischemic reperfusion in rats by inhibiting the expression of ICAM-1 and IL-18. Exp Ther Med 6: 1322-1326, 2013.

9. Zhang W, Guo Y, Yu S, Wei J and Jin J: Effects of edaravone on the expression of $\beta$-defensin- 2 mRNA in lung tissue of rats with myocardial ischemia reperfusion. Mol Med Rep 7: 1683-1687, 2013.

10. Alkan M, Celik A, Bilge M, Kiraz HA, Kip G, Ozer A, Sivgin V, Erdem O, Arslan M and Kavutcu M: The effect of levosimend an on lung damage after myocardial ischemia reperfusion in rats in which experimental diabetes was induced. J Surg Res 193: 920-925, 2015.

11. Yamamoto S, Kazama JJ and Fukagawa M: Autophagy: A two edged swordin diabetes mellitus. Biochem J 456: e1-e3, 2013.

12. Gao S, Jia JY, Yan TK, Yu YM, Shang WY, Wei L, Zheng ZF, Fang P, Chang BC and Lin S: Effects of ammonium pyrrolidine dithiocarbamate (PDTC) on osteopontin expression and autophagy in tubular cells in streptozotocin-induced diabetic nephropathy rat. Zhonghua Yi Xue Za Zhi 96: 3590-3595, 2016 (In Chinese).

13. Zhang J, Wang JS, Zheng ZK, Tang J, Fan K, Guo H and Wang JJ: Participation of autophagy in lung ischemia-reperfusion injury in vivo. J Surg Res 182: e79-e87, 2013.

14. Zhang D, Li C, Zhou J, Song Y, Fang X, Ou J, Li J and Bai C: Autophagy protects against ischemia/reperfusion-induced lung injury through alleviating blood-air barrier damage. J Heart Lung Transplant 34: 746-755, 2015.

15. Hong-Li S, Lei L, Lei S, Dan Z, De-Li D, Guo-Fen Q, Yan L, Wen-Feng $\mathrm{C}$ and Bao-Feng Y: Cardioprotective effects and underlying mechanisms of oxymatrine against ischemic myocardial injuries of rats. Phytother Res 22: 985-989, 2008.

16. Xiao TT, Wang YY, Zhang Y, Bai CH and Shen XC: Similar to spironolactone, oxymatrine is protective in aldosterone-induced cardiomyocyte injury via inhibition of calpain and apoptosis-inducing factor signaling. PLoS One 9: e88856, 2014.

17. Li RS, Yu CL and Jin XZ: Effect of oxymatrine on beating of cultured myocardial cells in vitro. Zhongguo Yao Li Xue Bao 10: 530-532, 1989 (In Chinese). 
18. Institute of Laboratory Animal Resources (US): National Research Council: National Academy Press, Washington, DC, 1996.

19. Liu R, Fang X, Meng C, Xing J, Liu J, Yang W, Li W and Zhou H: Lung inflation with hydrogen during the cold ischemia phase decreases lung graft injury in rats. Exp Biol Med (Maywood) 240: $1214-1222,2015$.

20. Kuma A, Komatsu M and Mizushima N: Autophagy-monitoring and autophagy-deficient mice. Autophagy 13: 1619-1628, 2017.

21. Di Marco E, Jha JC, Sharma A, Wilkinson-Berka JL, Jandeleit-Dahm KA and de Haan JB: Are reactive oxygen species still the basis for diabetic complications? Clin Sci (Lond) 129: 199-216, 2015.

22. Liu J, Li S and Sun D: Calcium dobesilate and micro-vascular diseases. Life Sci 221: 348-353, 2019.

23. Flemming NB, Gallo LA, Ward MS and Forbes JM: Tapping into mitochondria to find novel targets for diabetes complications. Curr Drug Targets 17: 1341-1349, 2016.

24. Tai H, Wang MY, Zhao YP, Li LB, Jiang XL, Dong Z, Lv XN, Liu J, Dong QY, Liu XG and Kuang JS: Pulmonary function and retrobulbar hemodynamics in subjects with type 2 diabetes mellitus. Respir Care 62: 602-614, 2017.

25. Strojek K, Raz I, Jermendy G, Gitt AK, Liu R, Zhang Q, Jacober SJ and Milicevic Z: Factors associated with cardiovascular events in patients with type 2 diabetes and acute myocardial infarction. J Clin Endocrinol Metab 101: 243-253, 2016.

26. Barbarash O, Gruzdeva O, Uchasova E, Belik E, Dyleva Y and Karetnikova V: Biochemical markers of type 2 diabetes as a late complication of myocardial infarction: A case-control study. Arch Med Sci 13: 311-320, 2017.

27. Khatib D, Boettcher BT, Freed JK and Pagel PS: Acute, severe pulmonary arterial hypertension during off-pump coronary artery surgery: Is new myocardial ischemia, cardiac repositioning, or external mitral valve compression the culprit? J Cardiothorac Vasc Anesth 30: 1744-1747, 2016.

28. Evlakhov VI and Poiasov IZ: Pulmonary hemodynamics following experimental myocardial ischemia after the blockade of adrenergic receptors. Ross Fiziol Zh Im I M Sechenova 101: 44-53, 2015 (In Russian).

29. Wang M, Verhaegh R, Tsagakis K, Brencher L, Zwanziger D, Jakob HG, Groot H and Dohle DS: Impact of acute intestinal ischemia and reperfusion injury on hemodynamics and remote organs in a rat model. Thorac Cardiovasc Surg 66: 99-108, 2018.
30. Palladini G, Ferrigno A, Rizzo V, Tarantola E, Bertone V, Freitas I, Perlini S, Richelmi P and Vairetti M: Lung matrix metalloproteinase activation following partial hepatic ischemia/reperfusion injury in rats. ScientificWorldJournal 2014: 867548, 2014.

31. Luo W, Jin Y, Wu G, Zhu W, Qian Y, Zhang Y, Li J, Zhu A and Liang G: Blockage of ROS and MAPKs-mediated inflammation via restoring SIRT1 by a new compound LF10 prevents type 1 diabetic cardiomyopathy. Toxicol Appl Pharmacol 370: 24-35, 2019.

32. Zhang F, Yang F, Zhao H and An Y: Curcumin alleviates lung injury in diabetic rats by inhibiting nuclear factor- $\kappa \mathrm{B}$ pathway. Clin Exp Pharmacol Physiol 42: 956-963, 2015.

33. Conklin DJ, Guo Y, Jagatheesan G, Kilfoil PJ, Haberzettl P, Hill BG, Baba SP, Guo L, Wetzelberger K, Obal D, et al: Genetic deficiency of glutathione S-transferase $p$ increases myocardialsensitivity to ischemia-reperfusion injury. Circ Res 117: 437-449, 2015

34. Dehdashtian E, Mehrzadi S, Yousefi B, Hosseinzadeh A, Reiter RJ, Safa M, Ghaznavi H and Naseripour M: Diabetic retinopathy pathogenesis and the ameliorating effects of melatonin; involvement of autophagy, inflammation and oxidative stress. Life Sci 193: 20-33, 2018.

35. Montane J, Cadavez L and Novials A: Stress and the inflammatory process: A major cause of pancreatic cell death in type 2 diabetes. Diabetes Metab Syndr Obes 7: 25-34, 2014.

36. Barlow AD and Thomas DC: Autophagy in diabetes: $\beta$-cell dysfunction, insulin resistance, and complications. DNA Cell Biol 34: 252-260, 2015.

37. Chen X, Wu JX, You XJ, Zhu HW, Wei JL and Xu MY: Cold ischemia-induced autophagy in rat lung tissue. Mol Med Rep 11: 2513-2519, 2015.

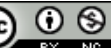

This work is licensed under a Creative Commons Attribution 4.0 International (CC BY-NC 4.0) License 